\title{
Validez de la escala breve de Zung para tamizaje del episodio depresivo mayor en la población general de Bucaramanga, Colombia
}

\begin{abstract}
Adalberto Campo, Luis Alfonso Díaz, Germán Eduardo Rueda
Grupo de Neuropsiquiatría, Facultad de Medicina, Universidad Autónoma de Bucaramanga, Bucaramanga, Colombia.

Introducción. Las escalas breves para identificar trastornos depresivos conservan la utilidad de las escalas extensas como instrumentos para tamizaje. Sin embargo, no se cuenta con una escala de estas características validada en población general colombiana.

Objetivo. Diseñar una escala abreviada de la escala de Zung para depresión para tamizaje de episodio depresivo mayor en adultos residentes en la comunidad general.

Materiales y métodos. A partir de la aplicación de la escala de Zung de veinte items se tomaron los diez items que mostraron la mayor correlación con la puntuación total. A estos items escogidos se les determinó la validez de constructo y la validez de criterio.

Resultados. Los diez items escogidos mostraron una consistencia interna de 0,803 , un único factor principal que explicaba el $36,6 \%$ de la varianza y sensibilidad de 95,5\%, especificidad de 70,3\%, kappa media de Cohen de 0,415 y 0,898 de área bajo la curva receptor-operador. Conclusiones. La escala abreviada de Zung muestra propiedades psicométricas similares a la escala extensa que permiten la utilización como instrumento de tamizaje en población adulta general.
\end{abstract}

Palabras clave: estudios de validación, tamizaje masivo, población urbana, adulto.

Validity of the brief Zung's scale for screening major depressive episode among the general population from Bucaramanga, Colombia

Introduction. Brief scales for identiying depressive disorder are as useful as long scales for screening. However, a validated scale with these characteristics is not avalaible in Colombia. Objective. To design a brief Zung's self-rating depression scale in order to screen major depressive episodes among adults dwelling in the general community.

Materials and methods. After filling-out the 20-item Zung's self-rating depression scale, the ten items with the higher correlation with total score were selected. Construct and criterion validity were computed for these ten items.

Results. The ten chosen items showed an internal consistency of 0,803 , one factor that accounted for $36,6 \%$ of the variance; sensitivity was $95,5 \%$; specificity, $70,3 \%$; Cohen's kappa, 0,415 ; and area under receptor-operator curve, 0,898.

Conclusions. The brief Zung's self-rating depression scale exhibits psychometric properties similar to the long version. This brief scale can be used as a screening device in the general population.

Key words: validation studies, mass screening, urban population, adult.

\section{$\overline{\text { Correspondencia: }}$}

Adalberto Campo, Facultad de Medicina, Universidad Autónoma de Bucaramanga, Calle 157 No 19-55, Cañaveral Parque, Bucaramanga, Colombia.

acampoar@unab.edu.co,neuropsiquia@unab.edu.co.

Recibido: 28/09/05; aceptado: 06/07/06
La categoría diagnóstica de trastornos depresivos incluye el trastorno depresivo grave, el trastorno distímico, los trastornos depresivos no específicos (trastorno depresivo leve, trastorno depresivo leve recurrente y trastorno disfórico premenstrual), así como los trastornos depresivos 
debidos a condiciones médicas o inducidos por el uso de sustancias (1). El trastorno depresivo mayor es el más prevalente en la población general colombiana. Sin embargo, la mayoría de las personas no consulta por estos síntomas $(2,3)$. De la pequeña proporción de personas que informa estas manifestaciones, un número reducido se identifica en la consulta médica general (4).

El trastorno depresivo mayor es el más discapacitante de los trastornos depresivos y deteriora en forma importante la calidad de vida, implica una alta carga de enfermedad (5), e incrementa significativamente el riesgo de presentar comorbilidad médica. Las personas deprimidas presentan, por ejemplo, una mayor frecuencia de enfermedades cardiovasculares que las no deprimidas (6), muestran una pobre adherencia a los tratamientos de enfermedades de larga evolución como la diabetes o la hipertensión arterial (este grupo de pacientes sigue con menor frecuencia las prescripciones médicas) (7) y se ausentan con más frecuencia de los sitios de trabajo, lo que tiene un impacto negativo en la economía de las naciones (8).

Las escalas son instrumentos útiles para la identificación de posibles casos de trastorno depresivo mayor en grandes poblaciones. Se dispone de varias escalas para el tamizaje (9). Sin embargo, en Colombia la escala de Zung para la depresión es la más usada porque no es necesario comprar los formatos (no está sujeta a derechos de autor) y es la más conocida, ya que se usó en los primeros estudios nacionales de salud mental por la facilidad al diligenciarla $(10,11)$.

La escala de Zung para la depresión es un instrumento que diligencia la propia persona y que consta de 20 componentes. Explora síntomas relacionados con la presencia de un episodio depresivo mayor y presenta un patrón de respuesta ordinal de cuatro opciones (12). Esta escala ha mostrado propiedades psicométricas aceptables en diferentes poblaciones clínicas y no clínicas (13-15). Sin embargo, se han propuesto versiones con un menor número de componentes. Estas versiones, que llamaremos abreviadas, se diseñaron con el propósito de tamizar el trastorno depresivo grave en adultos mayores. Se consideró que un número menor de componentes minimizaba el sesgo de respuesta relacionado con el cansancio que podía producir un instrumento de mayor longitud, y naturalmente reducía el tiempo dedicado a dar las respuestas (16-18).

Para abreviarlas incluso más, se ha propuesto el tamizaje con "escalas" de uno o dos componentes (19-21). Esta estrategia parece muy atractiva. No obstante, este tipo de instrumentos "ultrabreves" tiene varias limitaciones psicométricas. Primero, es muy poco factible que un único componente o una escala con menos de tres componentes abarque con suficiencia un constructo tan complejo como el concepto actual de episodio depresivo grave (22). Segundo, la confiabilidad de un solo componente es muy pobre si se compara con escalas con un número razonable de componentes (23).

Recientemente se propuso una versión abreviada de diez componentes de la escala de Zung para la depresión. Se observó que esta escala reducida conservaba las propiedades psicométricas (validez de constructo y de criterio) de la escala de veinte componentes. Sin embargo, esta versión se construyó a partir del patrón de respuesta de un número reducido de estudiantes universitarios, en su mayor porcentaje de sexo femenino (24). Es importante la validación en diferentes grupos poblacionales porque la validez y la confiabilidad de una escala varía según la población en que se usa (25).

Así mismo, es útil disponer de una escala abreviada con probada validez de constructo y de criterio para tamizar el trastorno depresivo más frecuente en la población general colombiana. Este es el primer paso para mejorar el proceso de identificación de posibles casos y, en consecuencia, aumentar el número de personas con tales síntomas adecuadamente tratadas, lo que podría reducir las consecuencias negativas asociadas con la presencia de este trastorno. Aunque la escala de Zung se diseñó para cuantificar la gravedad de un episodio depresivo en personas hospitalizadas, en personas residentes en la comunidad general sin diagnóstico formal de episodio depresivo mayor muestra un comportamiento y unas limitaciones 
similares a las que exhibe la escala para la depresión del Centro de Estudios Epidemiológico (CES-D) (9). Este último instrumento se diseñó para investigar el trastorno depresivo mayor en personas residentes en la comunidad (26).

En un par de informes precedentes se presentó la validez de constructo y de criterio de la escala de Zung en la población general colombiana (Campo-Arias A, Díaz-Martínez LA, Rueda-Jaimes GE. Factor analysis of the Zung's self-rating depresión scale in the general population from Bucaramanga, Colombia: a gender comparison. Enviado para publicación).

El objetivo de este trabajo fue validar (validez de constructo, validez de criterio y consistencia interna) una versión abreviada de diez componentes de la escala de Zung para la depresión en una muestra de adultos de la población general de Bucaramanga.

\section{Materiales y métodos}

Este estudio fue aprobado por el Comité de Ética de la Facultad de Medicina de la Universidad Autónoma de Bucaramanga. Todos los participantes dieron su consentimiento por escrito después de conocer y entender los objetivos del estudio.

\section{Muestreo}

Se tomó una muestra probabilística y representativa de 266 personas adultas de la población general residente en el área urbana de la ciudad de Bucaramanga con base en el censo de predios del Plan de Ordenamiento Territorial. Se excluyeron las personas iletradas. Se hacía un listado de las personas elegibles en cada hogar y posteriormente se elegía una mediante un listado de números aleatorios. Si la persona escogida al azar no se encontraba, se establecía una cita telefónicamente. El grupo estudiado estuvo conformado por 152 mujeres (57,1\%); la edad estaba comprendida entre los 18 y 65 años (promedio 37,4 años, $\mathrm{DE}=13,1$ ); el promedio de escolaridad formal fue de 9,8 (DE = 4,7) años; $52,2 \%$ eran trabajadores y $56,8 \%$ mantenían una relación de pareja estable (casados o en unión libre). Se seleccionaron al azar 355 predios con una reposición por posibles pérdidas del $20 \%$, de los cuales 26 se encontraron desocupados. Se excluyeron 14 personas por sus limitadas habilidades para la lectura y la escritura, 16 no fueron localizadas después de tres citas y 33 se negaron a participar.

\section{Tamaño de la muestra}

Considerando las recomendaciones de Chmura (28), se necesitaba una muestra mínima de 260 personas para una prevalencia actual de EDM de $15 \%$ basada en la prevalencia del episodio depresivo grave en la región (29); la sensibilidad esperada era de $90 \%$ y la especificidad, de $75 \%$.

\section{Medición de la escala de Zung y diagnóstico según el SCID I}

La escala de Zung para la depresión consta de 20 componentes que indagan la presencia de síntomas durante las dos semanas previas. Es una escala en la que cada componente presenta cuatro opciones de respuesta: nunca, a veces, casi siempre y siempre. A cada componente se le da una puntuación de uno a cuatro, $50,0 \%$ en sentido positivo y $50,0 \%$ en sentido inverso. La puntación total puede oscilar entre 20 y 80 con un punto de corte de 40 para los síntomas depresivos de importancia clínica (12). El rendimiento operativo de la escala con un punto de corte de 40 aparece publicado en otro artículo (27).

La aplicación de la escala de Zung y el diagnóstico definitivo de episodio depresivo mayor fueron realizados uno a continuación del otro de manera enmascarada e independiente por parte de dos profesionales entrenados y estandarizados para los efectos, quienes verificaron esto en el sitio de residencia del entrevistado.

Para los propósitos del presente estudio, el diagnóstico de episodio depresivo mayor con base en la entrevista estructurada del eje I del Manual Diagnóstico y Estadístico de la Asociación Psiquiátrica Americana (SCID-I) se estableció como el patrón de oro o estándar de referencia (30).

\section{Construcción de la escala abreviada}

Para escoger los componentes que formarían la nueva versión se fueron eliminando aquellos que tuviesen una correlación lineal de Pearson inferior a 0,5 con respecto a la puntuación total de la escala. A pesar de que esta medida tiene sus 
limitaciones para variables ordinales, muestra una relación importante con la "comunalidad" y el coeficiente de cada componente de una solución de factores; generalmente, los componentes que exhiben una baja correlación de Pearson presentan, igualmente, una pobre comunalidad y aportan muy poco a los factores $(23,31,32)$. Para cada versión con menor número de componentes se determinaba la curva de las características de receptor operador (ROC), y se la comparaba con la curva de la versión de 20 componentes mediante la prueba de DeLong, DeLong y Clarke-Pearson (33); si no era estadísticamente distinta, se procedía a eliminar el siguiente componente. Este procedimiento se realizó hasta que la diferencia fue significativa $(p<0,05)$, lo cual se presentó en la versión de nueve componentes, por lo que se adoptó la versión de diez componentes.

\section{Validación de la escala abreviada}

Validez de constructo. A los diez componentes elegidos se les determinó la consistencia interna mediante el coeficiente de alfa de Cronbach (34). Para validar el constructo, es decir, para conocer los posibles factores o dominios de la escala, se extrajeron los componentes principales. Este proceso se inició con la aplicación de la prueba de la esfericidad de Bartlett para establecer las características de la matriz de los coeficientes (35). Para estimar la correlación de los componentes con el constructo explorado se realizó la prueba de adecuación de la muestra de Kayser-Meyer-Olkin (36). Se verificó un análisis de factores por el método de componentes principales. Se definieron como factores principales los que mostraron valores propios mayores de 1,40 (esto para evitar el exceso de factores que se pueden identificar cuando se toma el criterio de $1,0[23,37])$, y estaban formados por un número mínimo de tres componentes después de la rotación oblicua en caso de ser necesaria. La rotación oblicua se aplicó porque se consideraba por anticipado que los síntomas que caracterizan un episodio depresivo grave están altamente relacionados (38). Se siguieron los mismos criterios para la selección de los componentes del estudio colombiano antes citado con el fin de tener una comparación más confiable (24).
Validez de criterio. Para escoger el mejor punto de corte para la escala abreviada se realizó una curva ROC (38). Se estimó el mejor punto de corte según la sensibilidad y la especificidad mostradas. A este punto se le calculó sensibilidad, especificidad, valores predictivos, razones de probabilidad y concordancia producto del azar (kappa media de Cohen) (39), e intervalos de confianza del 95\% (IC95\%) cuando estaba indicado.

Los datos se procesaron en el paquete estadístico STATA 9,0 (40).

\section{Resultados}

Con la entrevista clínica se identificaron 44 casos de episodio depresivo grave (16,5\%). La nueva versión quedó formada por los componentes que se presentan en el cuadro 1. El mejor punto de corte de la versión breve de diez componentes fue una puntuación de 20; en el cuadro 2 se muestran las propiedades psicométricas resultantes de la comparación de la escala breve con el patrón de oro.

La consistencia interna de esta versión fue de 0,803 y la de la versión original de 20 componentes, de 0,832. La prueba de esfericidad de Bartlett fue significativa $\left(\chi^{2}=628,897 ; g l=45\right.$, $p<0,001)$. La prueba de adecuación de la muestra de Kaiser-Meyer-Olkin fue excelente $(0,835)$. Un único factor relevante compone esta versión abreviada y explica el $36,6 \%$ de la varianza. Para la versión de 20 componentes se identificaron dos factores que explicaban 25,3 y $10,7 \%$ de la varianza, respectivamente. No fue necesario hacer rotación de los factores porque se extrajo un único factor principal. La solución y la matriz de factores se presentan en el cuadro 3 . No se observó diferencia significativa entre el área bajo la ROC entre la versión original $(0,901, \mathrm{IC} 95 \% 0,857$ a $0,945)$ y la versión breve $(0,898, I C 95 \% 0,852$ a $0,944 ; p=0,827)$. Estas curvas receptor operador aparecen en la figura 1.

\section{Discusión}

La escala abreviada de Zung para la depresión conserva todas las propiedades psicométricas de la escala original. El mejor punto de corte para estudios en la población general es de 20, y 
Cuadro 1. Componentes conservados con la numeración de la versión original y puntuación de la versión abreviada de Zung para depresión.

\begin{tabular}{|c|c|c|c|c|}
\hline Ítem & Nunca & A veces & Muchas veces & Siempre \\
\hline 1. Se ha sentido triste y decaído & 1 & 2 & 3 & 4 \\
\hline 3. Ha tenido ganas de llorar o ha llorado & 1 & 2 & 3 & 4 \\
\hline 8. Ha tenido palpitaciones & 1 & 2 & 3 & 4 \\
\hline 9. Se ha sentido cansado sin razón aparente & 1 & 2 & 3 & 4 \\
\hline 10. Ha tenido su mente tan despejada como siempre & 4 & 3 & 2 & 1 \\
\hline 11. Le ha sido fácil hacer las cosas como antes & 4 & 3 & 2 & 1 \\
\hline 12. Se ha sentido inquieto, intranquilo & 1 & 2 & 3 & 4 \\
\hline 13. Se ha sentido más irritable que de costumbre & 1 & 2 & 3 & 4 \\
\hline 16. Ha disfrutado sus actividades cotidianas & 4 & 3 & 2 & 1 \\
\hline 19. Ha encontrado agradable vivir & 4 & 3 & 2 & 1 \\
\hline
\end{tabular}

Cuadro 2. Comparación con el patrón de oro en un punto de corte de 20.

\begin{tabular}{|c|c|}
\hline Propiedad & Valor \\
\hline Sensibilidad & 95,5\% (IC95\% 83,3-99,2) \\
\hline Especificidad & $70,3 \%(I C 95 \% 63,7-76,1)$ \\
\hline Valor predictivo positivo & $38,9 \%(I C 95 \% 29,8-48,8)$ \\
\hline Valor predictivo negativo & $98,7 \%($ IC95\% 95,0-99,8) \\
\hline Razón de probabilidad positiva & 3,211 \\
\hline Razón de probabilidad negativa & 0,065 \\
\hline Kappa media de Cohen & 0,415 (IC95\% 0.409-0,421) \\
\hline
\end{tabular}

conserva una alta sensibilidad y una buena especificidad.

La escala abreviada de Zung propuesta en el presente estudio muestra una buena validez de constructo y de criterio. Similar a la versión corta presentada por Díaz et al., esta adaptación mostró una buena consistencia interna y un único factor importante que daba cuenta de algo más de un tercio de la varianza (24). Así mismo, estas versiones comparten nueve de los diez componentes, lo que sugiere que este grupo de componentes es el más sensible para identificar posibles casos de episodio depresivo mayor en la población general (31). Los componentes en discrepancia, "haber tenido palpitaciones" y "se ha sentido útil y necesario" (componente 8 en esta versión abreviada diseñada con base en los datos de una muestra de la población general, y componentes 15 en la versión propuesta a partir de los resultados en estudiantes universitarios, respectivamente), plantean la siguiente pregunta: ¿cuál de estos componentes se debería utilizar en próximas investigaciones o procesos de
Cuadro 3. Solución de factores de la escala abreviada de Zung para depresión.

\begin{tabular}{lcc}
\hline Ítem & Componente & Comunalidad \\
\hline Ánimo deprimido & 0,723 & 0,523 \\
Llanto fácil & 0,618 & 0,383 \\
Palpitaciones & 0,560 & 0,314 \\
Fatiga fácil & 0,555 & 0,307 \\
Concentración & 0,634 & 0,402 \\
Conación & 0,652 & 0,315 \\
Intranquilidad & 0,677 & 0,459 \\
Irritabilidad & 0,665 & 0,442 \\
Disfrute & 0,540 & 0,292 \\
Agradable vivir & 0,470 & 0,221 \\
Valor propio & 3,66 & - \\
\% de la varianza & 36,6 & - \\
\hline
\end{tabular}

tamizaje? Los datos sugieren que el componente 8 incorporado en la presente versión abreviada se comporta mejor en personas con un amplio rango de edad y una escolaridad promedio, tal como sucede en la población general, en tanto que el componente 15 incluido en la versión anterior de diez mostraría más sensibilidad en adultos jóvenes con mayor escolaridad. Es necesario confirmar esta hipótesis.

Sin duda esto corrobora que el desempeño de una escala y de un componente especial se ve fuertemente influenciando por las características de la población que diligencia el instrumento (25). En éste es importante resaltar que tanto la consistencia interna como el porcentaje de la varianza explicada por los factores fueron similares a los informados para la versión de 20 componentes (Campo-Arias A, Díaz-Martínez LA, Rueda-Jaimes GE. Factor analysis of the Zung's 


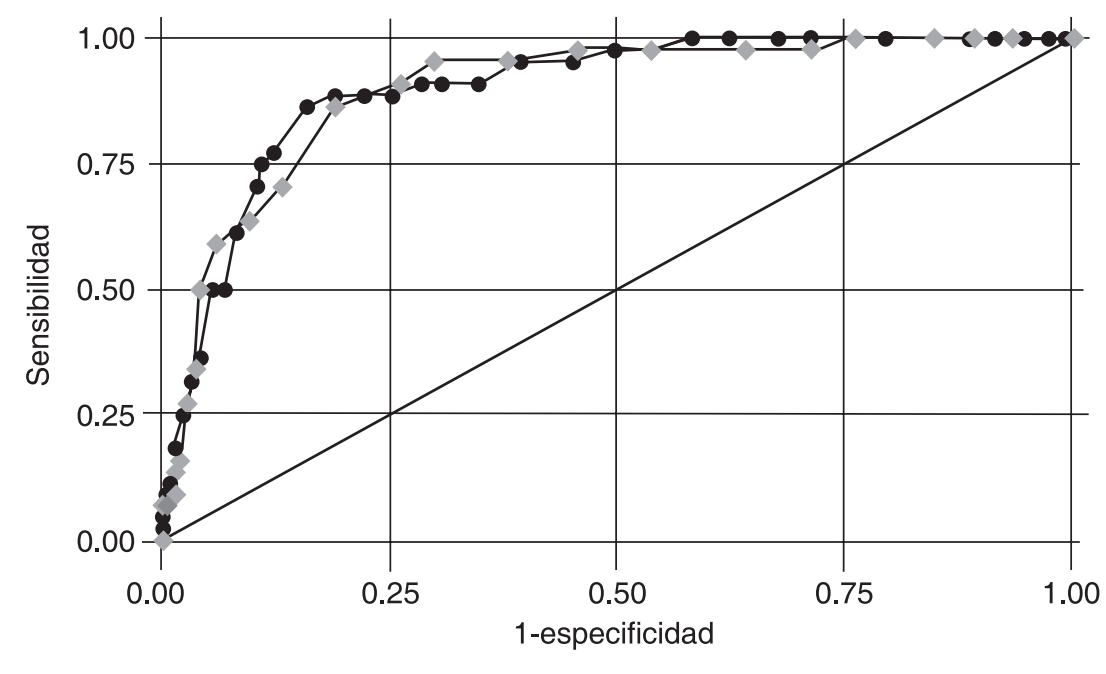

$\longrightarrow$ Zung original $\quad$ Zung breve

Figura 1. Curva de las características de receptor-operador $(\mathrm{ROC})$ de las escalas original y abreviada de Zung para la depresión en adultos de la población general de Bucaramanga, Colombia.

self-rating depression scale in the general population from Bucaramanga, Colombia: a gender comparison. Enviado para publicación). La consistencia interna de ambas versiones estuvo por encima de 0,80 . Se estima que este coeficiente es bueno cuando se encuentra entre 0,70 y 0,90 (41). No obstante, tal vez sea más diciente lo encontrado en la versión abreviada que se propone debido a que el valor de alfa de Cronbach es menos preciso cuando una escala tiene muchos componentes (por lo general, más de 20) (42). Con relación a los factores identificados, en las mejores condiciones se espera que éstos den cuenta de al menos el $50 \%$ de la varianza (23). No obstante, los factores tanto de la versión de 20 componentes como de la versión de 10 explican algo más del $35 \%$ de la varianza (CampoArias A, Díaz-Martínez LA, Rueda-Jaimes GE. Factor analysis of the Zung's self-rating depression scale in the general population from Bucaramanga, Colombia: a gender comparison. Enviado para publicación). Esto probablemente se deba a la baja "comunalidad", inferior a 0,30, mostrada por algunos componentes. Sin duda, los componentes excluidos presentaban valores inferiores. Esta situación se presenta cuando se extrae un único factor. Como la comunalidad de cada componente es igual a la suma de los cuadrados del componente de cada factor, ésta se reduce en forma dramática cuando se identifica un solo factor que explica menos del $50 \%$ de la varianza $(23,31)$. Cuando propuso la escala que lleva su nombre para determinar la gravedad de un episodio depresivo en personas hospitalizadas, Zung expuso que ésta abarcaba tres dominios, a saber, síntomas del estado de ánimo, síntomas cognoscitivos y síntomas vegetativos (12). El constructo actual de un episodio depresivo según la Asociación Psiquiátrica Americana está conformado por la presencia diaria y durante la mayor parte del día en el curso de al menos dos semanas de por lo menos uno de dos síntomas principales (estado de ánimo deprimido y disminución del interés por actividades que se consideran placenteras, o anhedonia) y de por lo menos otros cuatro síntomas secundarios 0 asociados (aumento o disminución de peso, insomnio o hipersomnio, agitación o entorpecimiento psicomotor, fatiga o pérdida de energía, ideas de minusvalía o de culpa excesiva, dificultad para concentrarse o indecisión y pensamientos de muerte o ideación o intento suicida) (1). La versión de 10 componentes presentada incluye los síntomas principales y la mayor parte de los 
síntomas considerados secundarios de un episodio depresivo mayor (1). Se conservan los síntomas que están presentes incluso en los episodios de gravedad leve a moderada. Síntomas más graves como las ideas recurrentes de muerte o el intento suicida se observan en los casos de mayor gravedad y se identifican con relativa facilidad con un instrumento de tamizaje o en una consulta médica habitual (43). El objeto del tamizaje en la depresión sería excluir a las personas no deprimidas y reconocer tempranamente los casos por debajo del umbral o leves en aquellas que tienen una prueba positiva (dado que aproximadamente sólo uno de cada tres positivos según la escala presentan realmente un episodio depresivo mayor), con el propósito de iniciar una intervención temprana para evitar los costos emocionales y sociales de un episodio depresivo de mayor gravedad (44).

Adicionalmente, esta versión conserva un número significativo de componentes con puntuación reversa. Esta característica de la puntuación es relevante para evitar el sesgo de marcar las respuestas siempre a la derecha (45).

Sin duda, un instrumento de menor número de componentes tiene mejor aceptación en la población general. El diligenciamiento será más fácil para personas con habilidades limitadas de lectoescritura, en personas con limitaciones cognoscitivas relacionadas con diferentes entidades clínicas no diagnosticadas en la comunidad general, y en individuos con escasa disposición de tiempo. Es necesario tener presente que la identificación de casos de trastorno depresivo grave en la población general tiene un impacto realmente significativo si ello incrementa en forma proporcional el número de personas adecuadamente tratadas por este trastorno (46).

Probablemente, las propiedades mostradas por la versión abreviada en esta población sólo puedan extrapolarse a la región andina colombiana. Las escalas presentar cambios significativos en las propiedades psicométricas relacionadas con factores culturales. De la misma forma, es prudente hacer algunas modificaciones lingüísticas a los componentes para adaptarlos a las características del lenguaje de cada región del país (47). Es dable que una parte de las diferencias encontradas en la prevalencia de síntomas depresivos en Colombia se deba más a un sesgo inducido por el instrumento que a diferencias reales explicadas por otros factores (genéticos o ambientales).

Además, es de suma importancia hacer ajustes en el punto de corte de la escala según la población estudiada. Algunas propiedades psicométricas se afectan muy desfavorablemente cuando la prevalencia del atributo o característica investigada presenta una baja prevalencia en la población estudiada (48-50).

Quedan por definir dos propiedades importantes para la evaluación de la confiabilidad de una prueba: primero, la reproducibilidad de la escala, sea la original o la abreviada, y segundo, la sensibilidad al cambio. Se conoce que la escala original de Zung tiene poca sensibilidad al cambio, por lo que no se recomienda para estudios de seguimiento (51). De tal suerte, se espera una alta correlación "prueba reprueba" con esta escala. En mujeres universitarias colombianas Campo et al. observaron que la escala de 20 componentes mostraba una excelente reproducibilidad $(r=$ $0,848)(52)$.

Se concluye que la escala abreviada de Zung para la depresión es un instrumento con una alta sensibilidad, una buena especificidad, una excelente consistencia interna y una importante homogeneidad. Es mandatorio ajustar el punto de corte para cada población. Se necesita verificar las propiedades psicométricas de esta versión abreviada en otras regiones colombianas y en otros países.

Esta escala abreviada y validada, con el rendimiento establecido, determina que en condiciones de baja prevalencia, un puntaje inferior a 20 puntos virtualmente descarta la existencia de un episodio depresivo mayor.

\section{Conflicto de intereses}

Ningún conflicto de intereses que declarar.

\section{Financiación}

Este trabajo lo financió la Dirección de Investigaciones de la Universidad Autónoma de 
Bucaramanga (Código 2105). Actualmente, Adalberto Campo trabaja además con la Universidad de Santander, la Universidad del Sinú en Cartagena y la Universidad de Cartagena.

\section{Referencias}

1. American Psychiatric Association. Diagnostic and statistical manual of mental disorders. Fourth Edition. Washington DC: American Psychiatric Association; 2000.

2. Posada-Villa JA, Trevisi C. Prevalencia, severidad y necesidades no satisfechas del tratamiento de los trastornos de ansiedad, relacionados con sustancias, del estado de ánimo y del control de impulsos en adultos según el Estudio Nacional de Salud Mental, Colombia, 2003. Medunab 2004;7:65-72.

3. Posada-Villa JA, Aguilar-Gaxiola SA, Magaña CG, Gómez LC. Prevalencia de trastornos mentales y uso de servicios: resultados preliminares del Estudio Nacional de Salud Mental, Colombia, 2003. Rev Colomb Psiquiatr 2004;33:241-62.

4. Rodríguez CE, Puerta G. Prevalencia de síntomas de depresión y ansiedad en los pacientes de la consulta no psiquiátrica. Rev Colomb Psiquiatr 1997;26:273-88.

5. Üstün TB, Ayuso-Mateos L, Chatterji S, Mathers C, Murray CJ. Global burden of depressive disorders in the year 2000. Br J Psychiatry 2004;184:386-92.

6. Allonier C, Chevalier A, Zins M, Catelinois $\mathbf{O}$, Consoli SM, Lahon G. Anxiety or depressive disorders and risk of ischaemic heart disease among French power company employees. Int $\mathrm{J}$ Epidemiol 2004;33:779-86.

7. Nothwehr F, Stump T. Health-promoting behaviors among adults with type 2 diabetes: findings from the Health and Retirement Study. Prev Med 2000;30:407-14.

8. Thomas CM, Morris S. Cost of depression among adults in England in 2000. Br J Psychiatry 2003;183:514-9.

9. Pignone MP, Gaynes BN, Rushton JL, Burchell CM, Orleans CT, Mulrow CD, et al. Screening for depression in adults: a summary of the evidence for the U.S. Preventive Services Task Force. Ann Intern Med 2002;136:765-76.

10. Torres Y, Murelle L. Estudio Nacional sobre Alcoholismo y Consumo de Sustancias PsicoactivasColombia 1987. Medellín: Editorial Universidad de Antioquia; 1988.

11. Torres Y, Posada J. Estudio Nacional de Salud Mental y Consumo de Substancias Psicoactivas. Bogotá: Ministerio de Salud; 1993.

12. Zung WW. A self-report depression scale. Arch Gen Psychiatry 1965;12:63-70.
13. Raft D, Spencer RF, Toomey T, Brogan D. Depression in medical outpatients: use of the Zung scale. Dis Nerv Syst 1977;38:999-1004.

14. Chida F, Kayama A, Nishi N, Sakai A. Factor analysis of Zung Scale scores in a Japanese general population. Psychiatry Clin Neurosci 2004;58:420-6.

15. Kitamura T, Hirano H, Chen Z, Hirata M. Factor structure of the Zung Self-rating Depression Scale in first-year university students in Japan. Psychiatry Res 2004;128:281-7.

16. Tucker MA, Ogle SJ, Davidson JG, Eilenberg MD. Validation of a brief screening test for depression in the elderly. Age Ageing 1987;16:139-44.

17. Irwin M, Artin KH, Oxman MN. Screening for depression in the older adult. Criterion validity of the 10-item Center for Epidemiological Studies Depression Scale (CES-D). Arch Intern Med 1999;159:1701-4.

18. Lelito RH, Palumbo LO, Hanley M. Psychometric evaluation of a brief geriatric depression screen. Aging Ment Health 2001;5:387-93.

19. Chochinov HM, Wilson KG, Enns M, Lander S. "Are you depressed?" Screening for depression in the terminally ill. Am J Psychiatry 1997;154:674-6.

20. Mallon L, Hetta J. Detección de la depresión mediante cuestionarios: comparación de una sola pregunta con los datos de la entrevista en una muestra de adultos de edad avanzada. Eur J Psychiatry 2002;16:139-48.

21. Arroll B, Khin N, Kerse N. Screening for depression in primary care with two verbally asked questions: cross sectional study. BMJ 2003;327:1144-6.

22. Jaju A, Crask MR. The perfect design: optimization between reliability, validity, redundancy in scale items and response rates. Am Market Assoc 1999;10:127-31.

23. Streiner DL. Figuring out factors: the use and misuse of factor analysis. Can J Psychiatry 1994;39:135-40.

24. Díaz LA, Campo A, Rueda GE, Barros JA. Propuesta de una versión abreviada de la escala de Zung. Colomb Med 2005;36:168-72.

25. Blacker D, Endicott J. Psychometric properties: concepts of reliability and validity. En: Rush AJ, Pincus HA, First MB, Zarin DA, Blacker D, Endicott J et al. Handbook of psychiatric measures. Washington D.C.: American Psychiatric Association; 2002 (CD-ROM).

26. Radloff LS. The CES-D scale: a self-report depression scale for research in the general population. Appl Psychol Meas 1977;1:385-401.

27. Campo-Arias A, Díaz-Martínez LA, Rueda-Jaimes GE, Cadena LP, Hernández NL. Validation of Zung's self-rating depression scale among the Colombian general population. Soc Behav Pers 2006;34:87-94.

28. Chmura H. Evaluating medical test. Objective and quantitative guidelines. Newbury Park: Sage Publications Inc; 1992. 
29. Gómez-Restrepo C, Bohórquez A, Pinto D, Gill JF, Rondón M, Díaz-Granados N. Prevalencia de depresión y factores asociados con ella en la población colombiana. Rev Panam Salud Pública 2004;16:37886.

30. First MB, Spitzer RL, Gibbon M, Williams JB. Entrevista clínica estructurada para los trastornos de eje I del DSM-IV (versión clínica) SCID-I. Barcelona: Masson; 1999.

31. Ferré L. Selection of components in principal component analysis: a comparison of methods. Comput Stat Data Anal 1995;19:669-82.

32. Hogarty KY, Hines CV, Kromey JD, Ferron JM, Mumford KR. The quality of factor solutions in exploratory factor analysis: the influence of sample size, communality, and overdetermination. Educ Psychol Meas 2005;65:202-26.

33. DeLong ER, DeLong DM, Clarke-Pearson DL. Comparing the areas under two or more correlated receiver operating curves: a nonparametric approach. Biometrics 1988;44:837-45.

34. Cronbach LJ. Coefficient alpha and the internal structure of test. Psychometrika 1951;16:297-334.

35. Norman GR, Streiner DL. Bioestadística. Madrid: Mosby/Doyma Libros; 1996. p.129-48.

36. Kaiser HF. An index of factorial simplicity. Psychometrika 1974;39:31-6.

37. Gorsuch RL. Exploratory factor analysis: its role in item analysis. J Pers Asses 1997;68:532-60.

38. Rey JM, Morris-Yates A, Stanislaw H. Measuring the accuracy of diagnostic test using receiver operating characteristics (ROC) analysis. Int J Method Psychiatr Res 1992;2:39-50.

39. Cohen J. A coefficient of agreement for nominal scales. Educ Psychol Meas 1960;20:37-46.

40. Stata Corporation. STATA for windows 9.0. College Station: StataCorp LP; 2005.

41. Sánchez R, Gómez C. Conceptos básicos sobre validación de escalas. Rev Colomb Psiquiatr 1998;27:121-30.
42. Cortina JM. What is coefficient alpha? An examination of theory and applications. J Appl Psychol 1993;78:98104.

43. Nease DE Jr, Aikens JE. DSM depression and anxiety criteria and severity of symptoms in primary care: cross sectional study. BMJ 2003;327:1030-1.

44. Kessler D, Bennewith O, Lewis G, Sharp D. Detection of depression and anxiety in primary care: follow up study. BMJ 2002;325:1016-7.

45. Spector P. Summated rating scale construction. Thousands Oaks, CA: Sage Publications; 1992.

46. Kroenke K. Depression screening is not enough. Ann Intern Med 2001;134:418-20.

47. Rubio-Stipec M, Hicks MH, Tsuang MT. Cultural factors influencing the selection, use, and interpretation of psychiatric measures. En: Rush AJ, Pincus HA, First MB, Zarin DA, Blacker D, Endicott J et al. Handbook of psychiatric measures. Washington D.C.: American Psychiatric Association; 2002 (CD-ROM).

48. Byrt T, Bishop J, Carlin JB. Bias, prevalence and kappa. J Clin Epidemiol 1993;46:423-9.

49. Streiner DL. Diagnosing tests: using and misusing diagnostic and screening tests. J Person Assess 2003;81:209-19.

50. Whiting P, Rutjes AW, Reitsma JB, Glas AS, Bossuyt PM, Kleijnen J. Sources of variation and bias in studies of diagnostic accuracy. A systematic review. Ann Intern Med 2004;140:189-202.

51. Zung WW. Zung self-rating depression scale (Zung SDS). En: Rush AJ, Pincus HA, First MB, Zarin DA, Blacker D, Endicott $\mathrm{J}$ et al. Handbook of psychiatric measures. Washington D.C.: American Psychiatric Association; 2002 (CD-ROM).

52. Campo-Arias A, Díaz-Martínez LA, Rueda-Jaimes GE, Barros-Bermúdez JA. Validación de la escala de Zung para depresión en universitarias de Bucaramanga, Colombia. Rev Colomb Psiquiatr 2005;34:54-62. 